\title{
HEATING A THERMOELASTIC HALF SPACE WITH A SURFACE ABSORPTION PULSED LASER USING FRACTIONAL ORDER THEORY OF THERMOELASTICITY
}

\author{
ISMAIL M. TAYEL \\ Department of Mathematics, College of Education, Majmaah University, Al-Majmaah, Saudi Arabia \\ e-mail: i.tayel@mu.edu.sa
}

AMIN F. HASSAN

Department of Physics, Faculty of Science, Helewan University, Cairo, Egypt

e-mail: aminhassan40@yahoo.com

\begin{abstract}
In this work, the problem of illuminating a thermoelastic half space by a laser beam is solved by utilizing the fractional order theory of thermoelasticity. The assumptions that the illuminated surface is exposed to a cooling effect and free from traction are considered. The problem is solved using Laplace transform techniques. The inverse Laplace transform has been calculated in numerical fashion. The obtained results are presented graphically.
\end{abstract}

Keywords: laser radiation, surface absorption, cooling effect, fractional order theory, thermoelasticity

\section{Introduction}

In the classical uncoupled theory of thermoelasticity, the heat equation is devoid of any terms symbolizing the elastic state of the body, which contradicts the truth that the variation in the elastic state produces thermal effects and vice versa. In addition, the heat equation built upon Fourier's law, and thus its nature being parabolic, leads to propagation of thermal waves with an infinite speed. According to physics, the previous facts may lead to inaccurate results, which prompted many of those interested in thermoelasticity to rectify the shortcomings of this theory. As a serious endeavor to address the flaws of the former theory, Biot (1956) derived the classical coupled theory of thermoelasticity (CTE). In Biot's theory, the coupling between strain fields and temperature was taken into account but its heat equation was still of a parabolic type, which was related to the reliance on Fourier's law.

Lord and Shulman (1967) proposed the generalized theory of thermoelasticity with one relaxation time by employing the Cattaneo (1948) approach. The energy equation of this theory was of a hyperbolic type, which implied a finite velocity for heat propagation and thus the weakness of Biot's model was tackled. Among the significant studies applied this theory were the proofs of uniqueness theorems by Ignaczak $(1979,1982)$ and Sherief $(1987)$. The analytical solution adequate for small times for an infinite elastic medium heated by a continuous heat source was obtained by Sherief (1986).

Recently, considerable proceeding has been detected in applications employing fractional calculus. In many branches of science, several mathematical models that describe physical phenomena have been adjusted by utilizing fractional calculus (Machado et al. (2013)). Caputo and Mainardi (1971a,b) and Caputo (1974) used fractional derivatives to the describe viscoelastic substances and did not observe a contradiction with the experimental results. A theoretical foundation for using fractional calculus in viscoelasticity was introduced by Bagly and Torvik (1983). 
In thermoelasticity, fractional calculus was utilized to modify the heat equation and then many models were introduced. Povstenko (2005) employed fractional derivatives to formulate the quasi-static uncoupled model of thermoelasticity. Povstenko (2009) did a survey study in thermoelasticity based on heat equations whose time and space derivatives were fractional. Youssef (2010) introduced the fractional order generalized thermoelasticity theory and applied this theory to a problem of an elastic semi-infinite medium exposed to a thermal shock. As a generalization to both coupled and generalized theories, Sherief et al. (2010) formulated the fractional order theory of thermoelasticity. Using Sherief's model, the solution of a thermoelasticity problem was successfully obtained for a semi-infinite medium possessing three different boundary conditions by applying the state space approach and the Laplace transform (Kothari and Mukhopadhyay, 2011). Sherief and Abd El-Latief (2013) solved a one-dimensional thermal shock problem for a semi-infinite body whose thermal conductivity was variable. They also applied the same theory to obtain a solution to a problem of a $1 \mathrm{D}$ semi-infinite medium by using various forms of functions representing the thermal shock. Raslan $(2014,2015,2016)$ applied Sherief's theory to study the thermoelasticity problem for different mediums and obtained some significant results.

When a solid is illuminated by a pulsed laser, a localized temperature increase due to absorbed power, which in turn produces thermal expansion and generates a thermoelastic wave. McDonald (1990) discussed generation of thermoelastic waves in solids created by laser illumination having a Gaussian profile. Ezzat et al. (2012) deduced an ultrafast fractional thermoelasticity model to study heat conduction in a thermoelastic thin metal film illuminated by a femtosecond laser pulse. Henain et al. (2014) studied the illumination of the surface of a thermoelastic 2D semi-infinite target by employing the Lord and Shulman theory. Allam and Tayel (2017) solved the problem of heating a thermoelastic non-homogeneous isotropic half space by a pulsed laser.

The purpose of this paper is to study the effect of the fractional parameter on a half-space illuminated uniformly by a laser beam and exposed to heat losses by employing the fractional order theory of thermoelasticity. The medium surface is taken as traction free. The copper material has been selected to obtain numerical results that are shown graphically.

\section{Problem formulation and basic equations}

Consider a homogeneous, isotropic thermoelastic semi-infinite target of density $\rho$, Lamé's constants $\lambda$ and $\mu$, thermal conductivity $k$, thermal relaxation time $\tau_{0}$, absorption coefficient $A_{0}$ and specific heat at constant strain $C_{E}$. The medium, occupying the region $z \geqslant 0$ and initially at uniform temperature $T_{0}$. The surface $z=0$ is uniformly illuminated by a laser beam and considered to be traction free, in addition it is exposed to heat losses. The Cartesian coordinates $(x, y, z)$ will be employed in the solution with the $z$-axis pointing vertically into the medium.

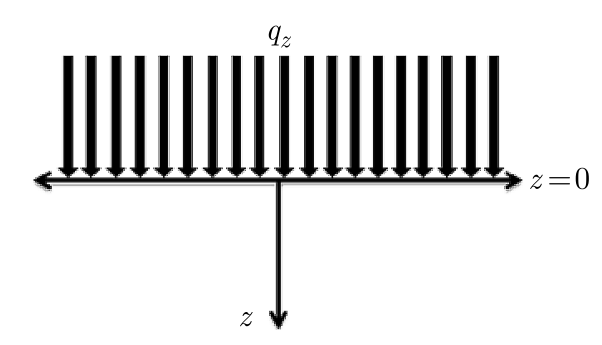

Fig. 1. Geometry of the target and heating process

The fundamental equations for a linear, homogeneous and isotropic thermoelastic body in the absence of body forces and heat source was derived by Sherief et al. (2010): 
— The energy equation

$$
k \nabla^{2} T=\left(1+\tau_{0} \frac{\partial^{\alpha}}{\partial t^{\alpha}}\right) \frac{\partial}{\partial t}\left(\rho C_{E} T+\gamma T_{0} e\right)
$$

where $T$ is the absolute temperature, $T_{0}$ is chosen such that $\left|\left(T-T_{0}\right) / T_{0}\right| \ll 1, \alpha$ is the order of the time fractional derivative in the Caputo sense, such that $0 \leqslant \alpha \leqslant 1, \gamma=(3 \lambda+2 \mu) \alpha_{T}$ and $\alpha_{T}$ is the coefficient of linear thermal expansion.

- The equation of motion

$$
\sigma_{i j, j}=\rho \frac{\partial^{2} u_{i}}{\partial t^{2}}
$$

where $\sigma_{i j}$ are components of the stress tensor and $u_{i}$ are components of the displacement vector.

The heating process is chosen to be a homogeneous illumination, and thus the problem will be a one-dimensional, which gives the displacement components

$$
u_{x}=0 \quad u_{y}=0 \quad u_{z}=w(z, t)
$$

Thus, the strain components take the form

$$
e_{z z}=\frac{\partial w}{\partial z} \quad e_{x x}=e_{y y}=e_{x y}=e_{x z}=e_{y z}=0
$$

The volume dilatation $e$ and the Laplace operator will be given by

$$
e=e_{z z}+e_{x x}+e_{y y}=\frac{\partial w(z, t)}{\partial z} \quad \nabla^{2}(\cdot)=\frac{\partial^{2}}{\partial z^{2}}(\cdot)
$$

The stress components become

$$
\begin{aligned}
& \sigma_{z z}=2 \mu \frac{\partial w}{\partial z}+\lambda e-\gamma\left(T-T_{0}\right) \quad \sigma_{x x}=\sigma_{y y}=\lambda e-\gamma\left(T-T_{0}\right) \\
& \sigma_{x y}=\sigma_{x z}=\sigma_{z y}=0
\end{aligned}
$$

Substituting Eqs. (2.6) into Eq. (2.2) and using (2.3), one gets

$$
(2 \mu+\lambda) \frac{\partial^{2} w}{\partial z^{2}}-\gamma \frac{\partial T}{\partial z}=\rho \frac{\partial^{2} w}{\partial t^{2}}
$$

Differentiating Eq. (2.7) with respect to $z$, we obtain

$$
(2 \mu+\lambda) \nabla^{2} e-\gamma \nabla^{2} T=\rho \frac{\partial^{2} e}{\partial t^{2}}
$$

The boundary conditions at the irradiated surface

$$
-\left.k \frac{\partial T}{\partial z}\right|_{z=0}=\left.\left(1+\tau_{0} \frac{\partial^{\alpha}}{\partial t^{\alpha}}\right) q_{z} \quad \sigma_{z z}\right|_{z=0}=0
$$

with $q_{z}=A_{0} q_{0} \phi(t)-h \theta(0, t)$ and $\theta=T-T_{0}$, where $q_{z}$ represents the heat flux in the $z$ direction, $q_{0}$ is the intensity of the laser beam, $\phi(t)$ is a function describing the laser pulse profile, $h$ is the heat transfer coefficient responsible for cooling the surface, and $\theta(0, t)$ is the surface temperature (Hassan et al., 1996). 
The initial conditions of the problem are

$$
\begin{aligned}
& \left.\theta(z, t)\right|_{t=0}=\left.\frac{\partial \theta(z, t)}{\partial t}\right|_{t=0}=\left.0 \quad w(z, t)\right|_{t=0}=\left.\frac{\partial w(z, t)}{\partial t}\right|_{t=0}=0 \\
& \left.\sigma_{i j}(z, t)\right|_{t=0}=\left.\frac{\partial \sigma_{i j}(z, t)}{\partial t}\right|_{t=0}=0
\end{aligned}
$$

The governing equations will take a more appropriate form by considering the following nondimensional variables

$$
\begin{aligned}
& \left(z^{*}, w^{*}\right)=c_{1} \eta(z, w) \quad \tau_{0}^{*}=c_{1}^{2 \alpha} \eta^{\alpha} \tau_{0} \quad t^{*}=c_{1}^{2} \eta t \quad h^{*}=\frac{h}{c_{1} \rho C_{E}} \\
& q_{0}^{*}=\frac{\gamma q_{0}}{(\lambda+2 \mu) c_{1} \rho C_{E}} \quad \sigma_{i j}^{*}=\frac{\sigma_{i j}}{\mu} \quad \theta^{*}=\frac{\gamma\left(T-T_{0}\right)}{\lambda+2 \mu}
\end{aligned}
$$

where

$$
c_{1}^{2}=\frac{\lambda+2 \mu}{\rho} \quad \eta=\frac{\rho C_{E}}{k}
$$

For simplicity of writing, we will drop the stars of all variables. Thus, Eqs. (2.1) and (2.8), will take the form

$$
\nabla^{2} \theta=\left(1+\tau_{0} \frac{\partial^{\alpha}}{\partial t^{\alpha}}\right) \frac{\partial}{\partial t}(\theta+\varepsilon e) \quad \nabla^{2} e-\nabla^{2} \theta=\frac{\partial^{2} e}{\partial t^{2}}
$$

Using Eq. (2.11), the stress components will be

$$
\sigma_{z z}=\beta^{2}(e-\theta) \quad \sigma_{x x}=\sigma_{y y}=g e-\beta^{2} \theta
$$

where

$$
\beta^{2}=\frac{\lambda+2 \mu}{\mu} \quad g=\frac{\lambda}{\mu} \quad \varepsilon=\frac{T_{0} \gamma^{2}}{\rho c_{E}(\lambda+2 \mu)}
$$

\section{Problem solution}

The basic equations and the initial conditions support the idea of seeking the solution using the Laplace transform technique defined as (Debnath and Bhatta, 2015)

$$
\bar{f}(z, s)=\int_{0}^{\infty} f(z, t) e^{-s t} d t
$$

Thus, Eq. (2.12) will take the form

$$
D^{2} \bar{\theta}=\left(s+\tau_{0} s^{\alpha+1}\right)(\bar{\theta}+\varepsilon \bar{e}) \quad\left(D^{2}-s^{2}\right) \bar{e}-D^{2} \bar{\theta}=0
$$

Eliminating $\bar{e}$ or $\bar{\theta}$ between Eqs. (3.2), we obtain

$$
\left(D^{4}-B D^{2}+C\right)\{\bar{\theta}, \bar{e}\}=0
$$

where

$$
B=s^{2}+\left(s+\tau_{0} s^{\alpha+1}\right)(\varepsilon+1) \quad C=s^{2}\left(s+\tau_{0} s^{\alpha+1}\right) \quad D=\frac{d}{d z}
$$


Equation (3.3) can be reformulated as

$$
\left(D^{2}-k_{1}^{2}\right)\left(D^{2}-k_{2}^{2}\right)\{\bar{\theta}, \bar{e}\}=0
$$

where $k_{i}^{2}(i=1,2)$ are the roots of the following auxiliary equation

$$
k^{4}-B k^{2}+C=0
$$

Solving Eqs. (3.4), we obtain

$$
\bar{\theta}=\sum_{i=1}^{2} D_{i} e^{-k_{i} z} \quad \bar{e}=\sum_{i=1}^{2} E_{i} e^{-k_{i} z}
$$

Substituting Eqs. (3.6) into Eq. (3.2) 1 yields

$$
E_{i}=\frac{k_{i}^{2}-s\left(1+\tau_{0} s^{\alpha}\right)}{\varepsilon s\left(1+\tau_{0} s^{\alpha}\right)} D_{i} \quad i=1,2
$$

Thus, Eqs. (3.6) take the form

$$
\bar{\theta}=\sum_{i=1}^{2} D_{i} e^{-k_{i} z} \quad \bar{e}=\sum_{i=1}^{2} \frac{k_{i}^{2}-s\left(1+\tau_{0} s^{\alpha}\right)}{\varepsilon s\left(1+\tau_{0} s^{\alpha}\right)} D_{i} e^{-k_{i} z}
$$

Using Eq. (2.5) $)_{1}$ and integrating (3.8) $)_{2}$ with respect to $z$, it follows

$$
\bar{w}(z, s)=\sum_{i=1}^{2}-\frac{k_{i}^{2}-s\left(1+\tau_{0} s^{\alpha}\right)}{\varepsilon s k_{i}\left(1+\tau_{0} s^{\alpha}\right)} D_{i} e^{-k_{i} z}
$$

Substituting by Eqs. (3.8) into Eqs. (2.13), after applying (3.1), we obtain

$$
\begin{aligned}
& \bar{\sigma}_{z z}=\beta^{2} \sum_{i=1}^{2}\left(\frac{k_{i}^{2}-s\left(1+\tau_{0} s^{\alpha}\right)}{\varepsilon s\left(1+\tau_{0} s^{\alpha}\right)}-1\right) D_{i} e^{-k_{i} z} \\
& \bar{\sigma}_{x x}=\bar{\sigma}_{y y}=\sum_{i=1}^{2}\left(\beta^{2} \frac{k_{i}^{2}-s\left(1+\tau_{0} s^{\alpha}\right)}{\varepsilon s\left(1+\tau_{0} s^{\alpha}\right)}-g\right) D_{i} e^{-k_{i} z}
\end{aligned}
$$

The boundary conditions after applying (2.11) and (3.1) become

$$
-\left.\frac{d \bar{\theta}}{d z}\right|_{z=0}=\left.\left(1+\tau_{0} s^{\alpha}\right) \bar{q}_{z} \quad \bar{\sigma}_{z z}\right|_{z=0}=0
$$

with $\bar{q}_{z}=A_{0} q_{0} \bar{\phi}(s)-h \bar{\theta}(0, s)$, where $\bar{\phi}(s)$ and $\bar{\theta}(0, s)$ are the laser profile and the surface temperature, respectively, in the transformed domain.

Substituting Eqs. $(3.8)_{1}$ and $(3.10)_{1}$ into Eqs. (3.11), one gets

$$
\sum_{i=1}^{2} k_{i} D_{i}=\left(1+\tau_{0} s^{\alpha}\right)\left(A_{0} q_{0} \bar{\phi}(s)-h \bar{\theta}(0, s)\right) \quad \sum_{i=1}^{2} f_{i}(s) D_{i}=0
$$

where

$$
f_{i}(s)=\frac{k_{i}^{2}-s\left(1+\tau_{0} s^{\alpha}\right)}{\varepsilon s\left(1+\tau_{0} s^{\alpha}\right)}-1 \quad i=1,2
$$


Thus, from Eqs. (3.12) we obtain

$$
\begin{aligned}
& D_{1}=\frac{f_{2}\left(1+s^{\alpha} \tau_{0}\right)\left[-A_{0} q_{0} \bar{\phi}(s)+\bar{\theta}(0, s) h\right]}{k_{1} f_{2}-k_{2} f_{1}} \\
& D_{2}=\frac{f_{1}\left(1+s^{\alpha} \tau_{0}\right)\left[-A_{0} q_{0} \bar{\phi}(s)+\bar{\theta}(0, s) h\right]}{-k_{1} f_{2}+k_{2} f_{1}}
\end{aligned}
$$

Finally, to obtain $\bar{\theta}(0, s)$ substituting $D_{1}$ and $D_{2}$ into Eq. $(3.8)_{1}$ and set $z=0$, one gets

$$
\bar{\theta}(0, s)=\frac{A_{0} q_{0} \bar{\phi}(s)\left(1+s^{\alpha} \tau_{0}\right)\left(f_{1}-f_{2}\right)}{f_{1}\left[h\left(1+s^{\alpha} \tau_{0}\right)-k_{2}\right]-f_{2}\left[h\left(1+s^{\alpha} \tau_{0}\right)-k_{1}\right]}
$$

\section{Inverse Laplace transformation}

Now we will execute an inverse transformation for temperature, displacement and thermal stresses. Due to intricacy of obtaining the physical variables in the time domain by analytical methods, we will utilize a numerical method represented in the Riemann-sum approximation by applying the formula

$$
f(t)=\frac{e^{k t}}{t}\left[\frac{1}{2} \bar{f}(k)+\operatorname{Re} \sum_{n=1}^{N}(-1)^{n} \bar{f}\left(k+\frac{\mathrm{i} n \pi}{t}\right)\right]
$$

where Re is the real part and $\mathrm{i}=\sqrt{-1}$ is the imaginary unit and $N$ is an adequately large integer indicating the number of terms. For faster convergence, many numerical experiments discussed the value of $k$ and showed that it would be satisfactory when: $k t=4.7$ (Tzou, 1995).

\section{Application and computation}

As an illustrative example, the computations have been carried out on a semi-infinite medium of the copper material, assuming the value of the relaxation time to be $\tau_{0}=75 \cdot 10^{-5} \mathrm{~s}$. We consider the surface of the medium to be illuminated by a laser pulse having the maximum density $q_{0}=10^{4} \mathrm{~W} / \mathrm{m}^{2}$, and the temporal profile takes the form of a Gaussian distribution

$$
\phi(t)=\exp \left[-\left(\frac{t-t_{0}}{p}\right)^{2}\right]
$$

where $t_{0}$ is the time at which the distribution $\phi(t)$ gives the maximum value, $p$ is the time at which $\phi(t)$ reduces to $1 / e$.

Now, the temperature, displacement and thermal stresses will be calculated considering the following parameters (Henain et al., 2014): $T_{0}=293 \mathrm{~K}, \rho=8954 \mathrm{~kg} / \mathrm{m}^{3}, \alpha_{T}=1.78 \cdot 10^{-5} \mathrm{~K}^{-1}$, $p=10^{-3} \mathrm{~s}, C_{E}=383.1 \mathrm{~J} /(\mathrm{kg} \mathrm{K}), h=200 \mathrm{~W} /\left(\mathrm{m}^{2} \mathrm{~K}\right), \mu=3.86 \cdot 10^{10} \mathrm{~kg} /\left(\mathrm{m} \mathrm{s}^{2}\right), \lambda=7.76$. $\left.10^{10} \mathrm{~kg} /(\mathrm{m} \mathrm{s})^{2}\right), t_{0}=3 \cdot 10^{-3} \mathrm{~s}, k=386 \mathrm{~W} /(\mathrm{m} \mathrm{K}), A_{0}=0.01$.

\section{Results and discussion}

Figure 2a represents in curve (a) the laser pulse profile whose maximum value is at $t=0.003$, and curves representing the temporal surface temperature calculated for $h=0$ and $\alpha=1,0.9$, $0.8,0.5$. It also includes a curve representing the temporal surface temperature of the CTE theory $\left(\tau_{0}=0\right)$. It is shown that the temperature firstly increases with time, which can be attributed to 
the increased absorbed energy, which overcompensates the heat losses due to heat conductivity inside the material. At the time at which the absorbed power equals the conducted one inside the material, the temperature attains its maximum value, this explains why the maximum has occurred at a later time in the cases $\alpha=1$ and $\alpha=0.5,0.8,0.9$. After reaching the maximum, the absorbed power cannot compensate the losses, which leads to a decreasing temperature. After switching off the laser pulse, the absolute value of the gradient of temperature becomes smaller than that during the laser pulse, which is due to the absence of laser radiation, which leads to a smaller gradient of temperature and, therefore, small conduction of the heat energy. It is also noted that for $\alpha=0.5,0.8,0.9$ the maximum of temperature occurs at a later time than in the case of $\alpha=1$, which can be attributed to the velocity of the heat waves which become faster with decreasing $\alpha$. Thus, it takes longer to compensate the heat losses by the conductivity and also gives the maximum value of the temperature smaller than $\alpha=1$. It is clearly noted that the curve of $\alpha=0.5$ coincides with the carve of CTE, which indicates that the curves $\alpha \in[0,1)$ follow the behavior of the CTE theory.

Figure $2 \mathrm{~b}$ represents in curve (a) the laser pulse profile whose maximum value is at $t=0.003$, and the other curves showing the temporal surface temperature calculated for $h \neq 0$ and $\alpha=1,0.5$. A very slight shift in the maximum temperature towards greater $t$ is seen in the figure. This is owing to the cooling effect which contributes together with conductivity of the material to support heat losses and thus the surface temperature approaching to zero at the end of the laser pulse. It is also noted a weak effect for the parameter $\alpha$ compared with Fig. 2a.

(a)

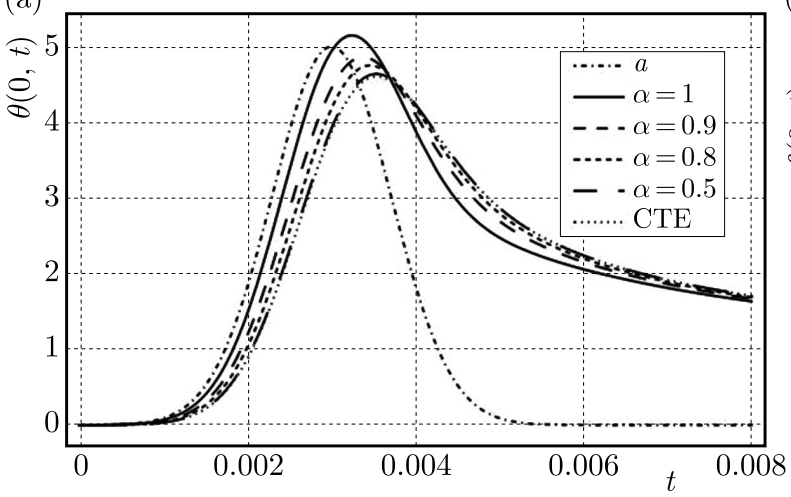

(b)

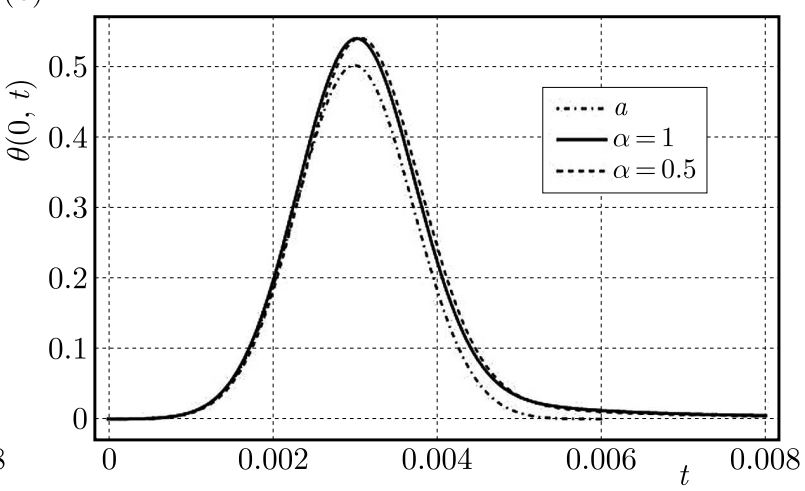

Fig. 2. The temporal profile of the laser pulse and the surface temperature calculated for: (a) $h=0$, (b) $h \neq 0$

(a)

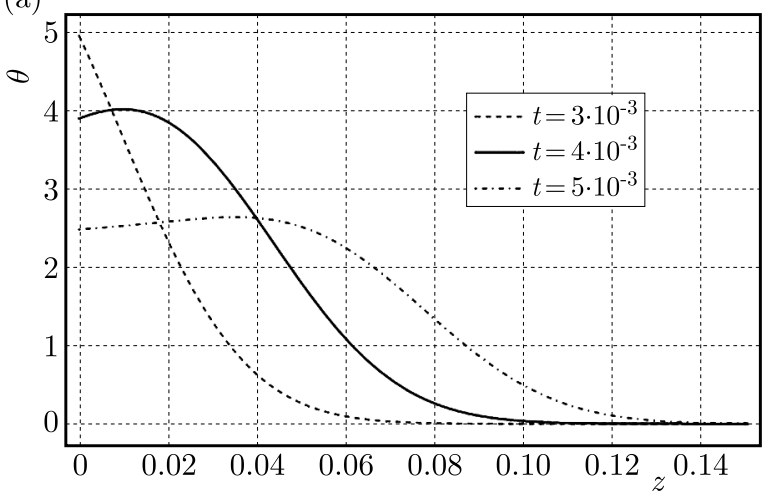

(b)

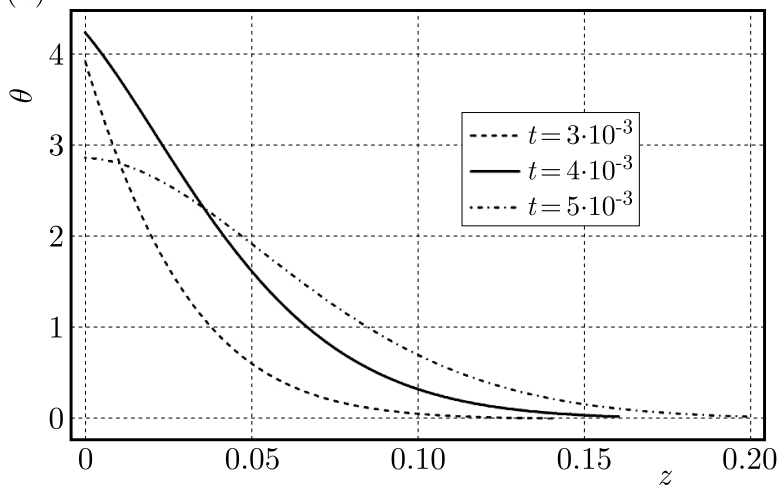

Fig. 3. The temperature $\theta$ calculated for $h=0$ and (a) $\alpha=1$, (b) $\alpha=0.5$

Figure 3a explains the spatial temperature $\theta$ computed for $\alpha=1, h=0$ at different times. The nature of the hyperbolic type as in the Cattaneo model is clearly seen, as the figure reveals 
a finite velocity expressing through the strong gradient at different locations. In the figure, the temperature has the maximum value at $t=3 \cdot 10^{-3}$, which can be illustrated in view of Fig. 2a, where the maximum value of the surface temperature is next to this time. It is also seen that the temperature moves deeper into the medium as the time increases.

Figure 3b explains the temperature $\theta$ versus $z$ computed for $\alpha=0.5, h=0$ at different times. As seen in the figure, the temperature at $t=4 \cdot 10^{-3}$ is greater than the that at $t=3 \cdot 10^{-3}$. This can be interpreted from Fig. $2 \mathrm{~b}$, where the maximum value of the surface temperature occurs near the time $\left(t=4 \cdot 10^{-3}\right)$. The figure reveals that $\theta$ is reduced in the vicinity of the irradiated surface and penetrates to greater $z$ values than the corresponding ones given in Fig. 3a. This behavior is owing to the infinite velocity, which characterizes the heat waves for $\alpha \in[0,1)$.

Figure 4a explains the temperature $\theta$ versus $z$ computed for $\alpha=1, h \neq 0$ at diverse times. The figure reveals that the maximum of $\theta$ is not on the illuminated surface, but it is shifted towards higher $z$ values. This behavior is due to the cooling effect on the illuminated surface and the laser power which is absorbed and transferred inside the target, causing an increase of the temperature. It is also seen a finite velocity for the temperature.

Figure $4 \mathrm{~b}$ explains the temperature $\theta$ versus $z$ computed for $\alpha=0.5, h \neq 0$ at diverse times. The figure displays the same behavior of Fig. 4a for the surface temperature, but it differs in the temperature penetrating to greater $z$ values at the same times, This is due to the infinite velocity of the heat waves.

(a)

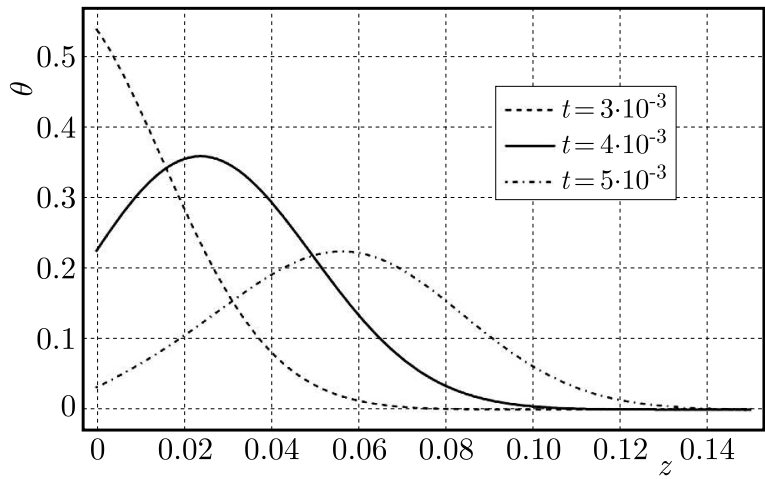

(b)

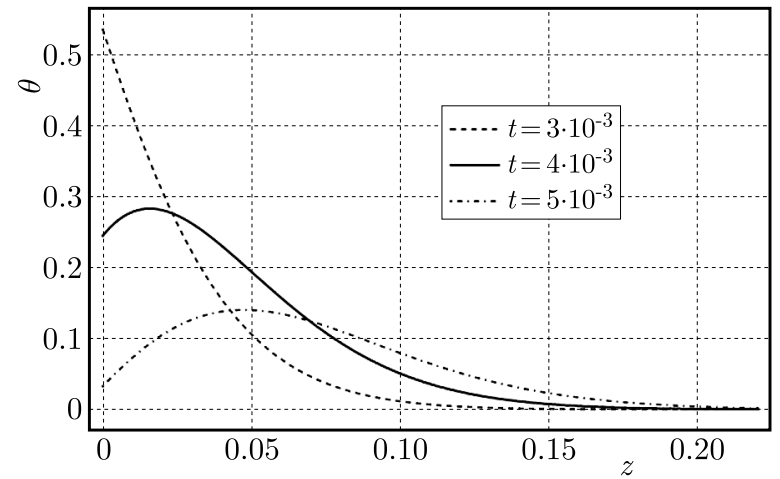

Fig. 4. The temperature $\theta$ calculated for: (a) $\alpha=1, h \neq 0$ and (b) $\alpha=0.5, h \neq 0$

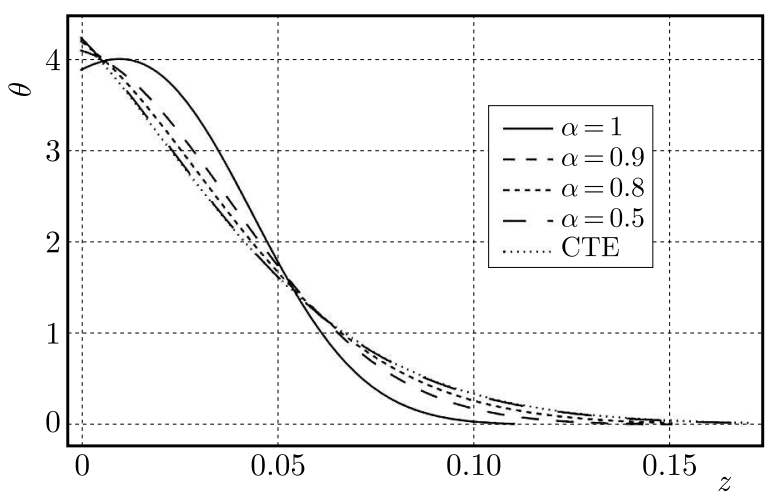

Fig. 5. The temperature $\theta$ with diverse values of $\alpha$ at $t=4 \cdot 10^{-3}$ and $h=0$

Figure 5 illustrates the temperature $\theta$ versus $z$ with diverse values of $\alpha$ at $t=4 \cdot 10^{-3}$ in the absence of cooling. The figure shows a clear effect of the parameter $\alpha$ on temperature where, for $\alpha=1$, the finite velocity of $\theta$ clearly appears. By decreasing the value of $\alpha$, the temperature penetrates to a great $z$ value into the target. This behavior is owing to the infinite velocity, 
which appears for $\alpha=0.5,0.8,0.9$. As in Figs. 2a and 3a, the curve of $\alpha=0.5$ coincides with the curve of the CTE theory, and for $0 \leqslant \alpha<1$ the curves follow the behavior of CTE theory.

Figure 6 illustrates the displacement $w$ versus $z$ computed for $\alpha=1, h=0$ at diverse times. It is seen that, the displacement is possess a negative values and located in the vicinity of the irradiated surface. Then, it moves towards greater $z$-value until vanishing, this is owing to the heating effect which allowed the expansion to occur in the upward direction while the positive $z$-axis is considered downwards. As $t$ increases, the displacement moves deeper inside the target.

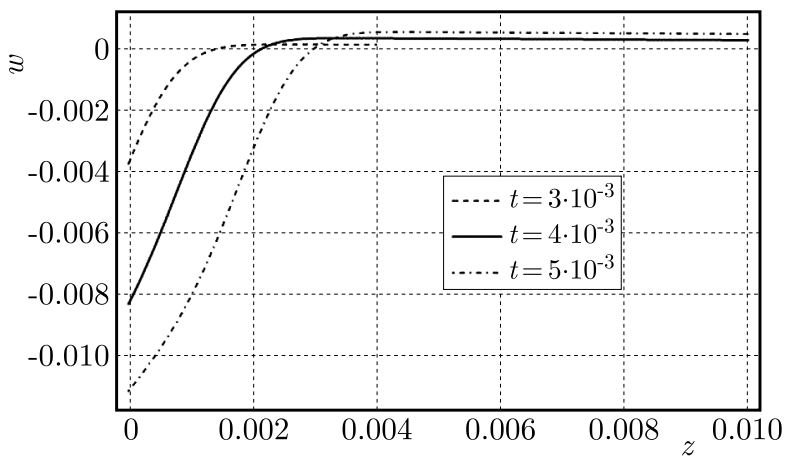

Fig. 6. The displacement $w$ calculated for $\alpha=1$ and $h=0$

Figure 7a illustrates the displacement $w$ versus $z$ computed for $h \neq 0$ at $t=4 \cdot 10^{-3}$ with the parameter $\alpha$. The figure reveals that the parameter $\alpha$ has a slight effect on $w$, which is owing to the cooling effect, which gives the temperature the same behavior for the two values $\alpha=1$, $\alpha=0.5$, and makes the expansion occur almost equally.

Figure 7b illustrates the displacement $w$ versus $z$ computed for $h=0$ at $t=4 \cdot 10^{-3}$ with $\alpha$ as the parameter. The figure displays that $\alpha$ have a clear effect on $w$ in the absence of cooling. For diverse $\alpha$ values, the displacement starts having different values at the irradiated surface and increasing for greater $\alpha$. This can be attributed to the heating effect which differs for the values $\alpha=1$ and $\alpha=0.5,0.8,0.9$, as seen in Fig. 5. The figure shows an exact coincidence between the curves of $\alpha=0.5$ and of the CTE theory.

(a)

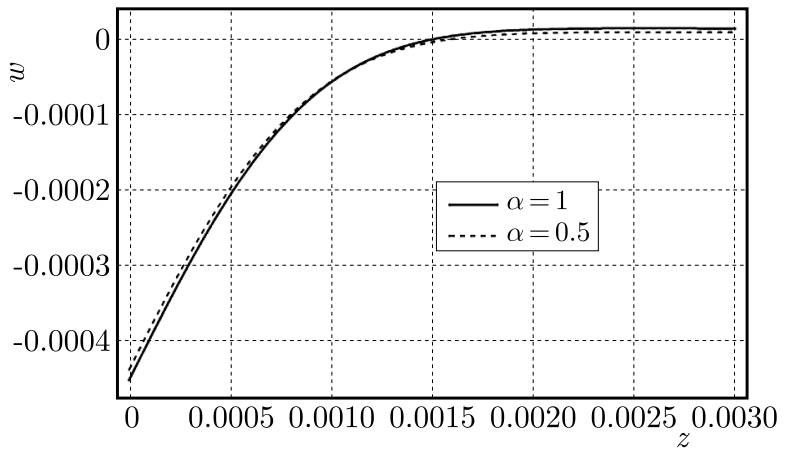

(b)

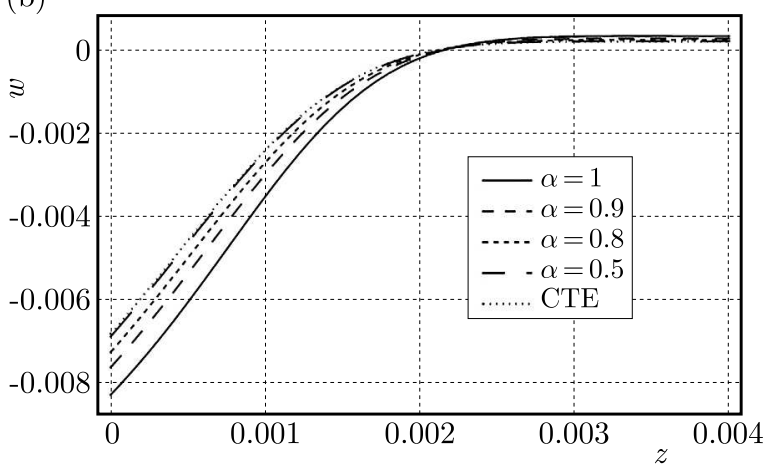

Fig. 7. The displacement $w$ with diverse values of $\alpha$ at $t=4 \cdot 10^{-3}$ and: (a) $h \neq 0$, (b) $h=0$

Figures 8a and 8b illustrate the stress $\sigma_{z z}$ computed for $h=0$ at diverse times for $\alpha=1$ and $\alpha=0.5$, respectively. The figures can be explained in view of Eq. (2.14) where, at a small time, $\theta$ has a greater effect than $e$ (gradient of $w$ ) and, therefore, the stress takes negative values. By increasing the time, $e$ becomes more effective near the illuminated surface, which grants the stress to take positive values. By penetrating more into the target, $e$ stops growing and temperature becomes more effective, which grants the stress to take negative values. It is noted 
that the stresses follow the behavior of temperature, and the curves display a clear effect for the parameter $\alpha$.

(a)

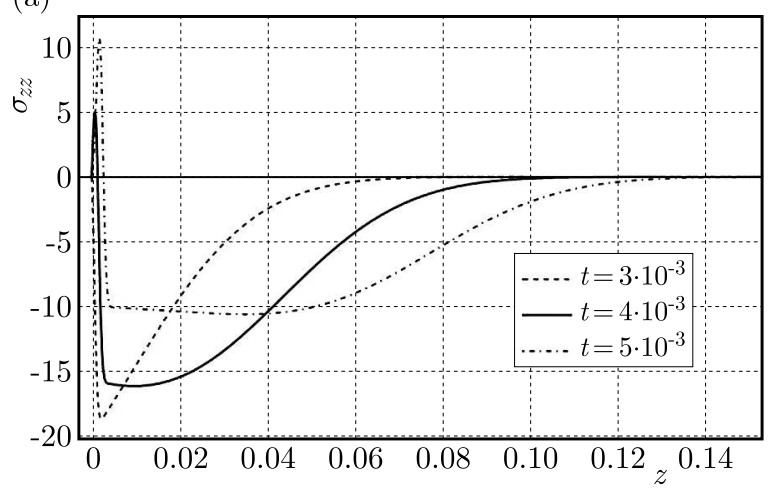

(b)

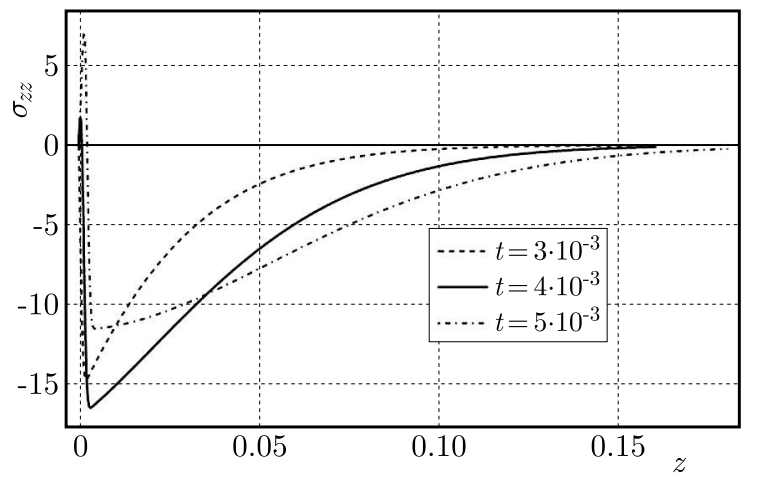

Fig. 8. The stress $\sigma_{z z}$ calculated for $h=0$ and (a) $\alpha=1$, (b) $\alpha=0.5$

Figures $9 \mathrm{a}$ and $9 \mathrm{~b}$ illustrate the stress $\sigma_{z z}$ computed for $h \neq 0$ at diverse times for $\alpha=1$ and $\alpha=0.5$, respectively. The curves display analogous behavior as in Figs. $8 \mathrm{a}$ and 8b, except that the values $\sigma_{z z}$ are much smaller than the corresponding ones in Figs. $7 \mathrm{~b}$ and $8 \mathrm{a}$, which is due to the cooling effect.

(a)

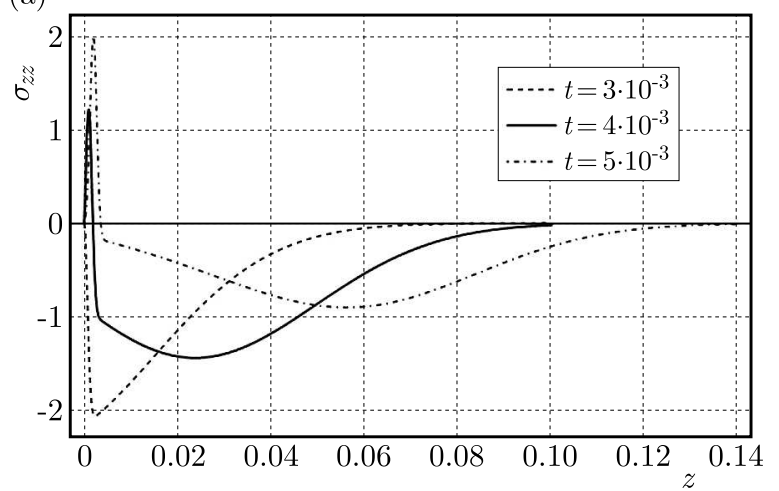

(b)

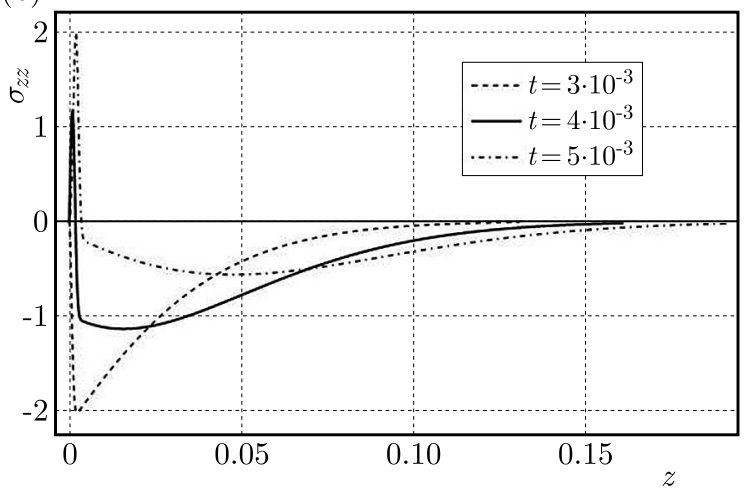

Fig. 9. The stress $\sigma_{z z}$ calculated for: (a) $\alpha=1$ and $h \neq 0$, (b) $\alpha=0.5$ and $h \neq 0$

Figure 10 illustrates the stress $\sigma_{z z}$ versus $z$ computed for $h=0$ at $t=4 \cdot 10^{-3}$ with $\alpha$ as the parameter. The figure shows a clear effect of $\alpha$ on the stresses, especially when following the behavior of the temperature.

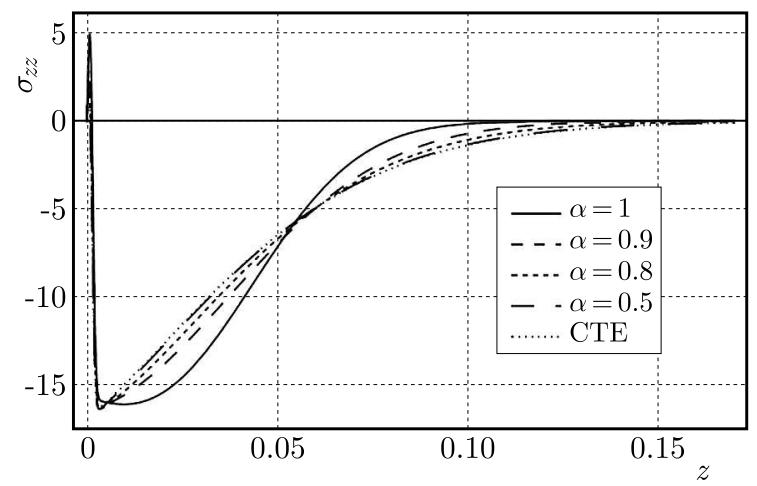

Fig. 10. The stress $\sigma_{z z}$ with diverse values of $\alpha$ at $t=4 \cdot 10^{-3}$ and $h=0$ 


\section{Conclusion}

In this paper, we discussed the problem of heating the surface of a homogeneous, isotropic thermoelastic semi-infinite medium by uniform illumination of a laser beam in the presence of the cooling effect. The problem has been solved using the fractional order theory of thermoelasticity. From the results discussed above, it can be concluded that:

- For $\alpha=1$, the waves propagate with a finite velocity representing a hyperbolic type as in the generalized theories of thermoelasticity, and do not contradict with the well-known physical behavior. For $\alpha \in[0,1)$, the waves propagate with an infinite velocity representing the classical coupled theory of thermoelasticity, which is more pronounced for $\alpha=0.5$.

- The parameter $\alpha$ has a clear effect on all variables in the absence of cooling and a slight effect in the presence of cooling.

- The cooling coefficient can play a main role in controlling the effect of the laser power.

Acknowledgment

The authors would like to thank Deanship of Scientific Research at Majmaah University for supporting this work under project number 1440/47.

\section{References}

1. Allam M.N.M., Tayel I.M., 2017, Generalized thermoelastic functionally graded half space under surface absorption of laser radiation, Journal of Theoretical and Applied Mechanics, 55, 1, $155-165$

2. Bagley R.L., Torvik P.J., 1983, A theoretical basis for the application of fractional calculus to viscoelasticity, Journal of Rheology, 27, 201-210

3. Biot M., 1956, Thermoelasticity and irreversible thermodynamics, Journal of Applied Physics, 27, 240-253

4. CAPuto M., 1974, Vibrations of an infinite viscoelastic layer with a dissipative memory, The Journal of the Acoustical Society of America, 56, 3, 897-904

5. Caputo M., Mainardi F., 1971a, A new dissipation model based on memory mechanism, Pure and Applied Geophysics, 91, 134-147

6. Caputo M., Mainardi F., 1971b, Linear models of dissipation in anelastic solids, La Rivista del Nuovo Cimento, 1, 2, 161-198

7. Cattaneo C., 1948, Sulla conduzione del calore, Atti del Seminario Matematico e Fisico dell' Universita di Modena, 3, 83-101

8. Debnath L., Bhatta D., 2015, Integral Transforms and their Applications, Taylor and Francis

9. Ezzat M.A., El-Karamany A.S., Fayik M.A., 2012, Fractional ultrafast laser-induced thermoelastic behavior in metal films, Journal of Thermal Stresses, 35, 7, 637-651

10. Hassan A., El-Nicklawy M.M., Nasr M.E., Hemida A.A., Abd El-Ghaffar A.O., 1996, Heating effects induced by a pulsed laser in a semi-infinite target in view of the theory of linear system, Journal of Optics and Laser Technology, 28, 337-343

11. Henain E.F., Hassan A.F., Megahed F., Tayel I.M., 2014, Thermoelastic half space under illumination of a laser beam using Lord and Shulman theory, Journal of Thermal Stresses, 37, $51-72$

12. IGNACZAK J., 1979, Uniqueness in generalized thermoelasticity, Journal of Thermal Stresses, 2, 2, $171-175$

13. IGNACZAK J., 1982, A note on uniqueness in thermoelasticity with one relaxation time, Journal of Thermal Stresses, 5, 257-263 
14. Kothari S., Mukhopadhyay S., 2011, A problem on elastic half space under fractional order theory of thermoelasticity, Journal of Thermal Stresses, 34, 7, 724-739

15. Lord H., Shulman Y., 1967, A generalized dynamical theory of thermo elasticity, Journal of the Mechanics Physics of Solids, 15, 299-309

16. Machado J., Galhano A., Trujillo J., 2013, Science metrics on fractional calculus development since 1966, Fractional Calculus and Applied Analysis, 16, 479-500

17. MCDonald F.A., 1990, On the precursor in laser-generated ultrasound waveforms in metals, Applied Physics Letters, 56, 3, 230-232

18. Povstenko Y.Z., 2005, Fractional heat conduction equation and associated thermal stress, Journal of Thermal Stresses, 28, 83-102

19. Povstenko Y.Z., 2009, Thermoelasticity that uses fractional heat conduction equation, Journal of Mathematical Sciences, 162, 2, 296-305

20. Raslan W., 2014, Application of fractional order theory of thermoelasticity to a 1D problem for a cylindrical cavity, Archives of Mechanics, 66, 4, 257-267

21. Raslan W., 2015, Application of fractional order theory of thermoelasticity in a thick plate under axisymmetric temperature distribution, Journal of Thermal Stresses, 38, 7, 733-734

22. Raslan W., 2016, Application of fractional order theory of thermoelasticity to 1D problem for spherical shell, Journal of Theoretical and Applied Mechanics, 54, 1, 295-304

23. Sherief H., 1986, Fundamental solution of the generalized thermoelastic problem for short times, Journal of Thermal Stresses, 9, 151-164

24. Sherief H., 1987, On uniqueness and stability in generalized thermoelasticity, Quarterly of Applied Mathematics, 45, 773-778

25. Sherief H., Abd El-Latief A.M., 2013, Effect of variable thermal conductivity on a half-space under the fractional order theory of thermoelasticity, International Journal of Mechanical Sciences, 74, 185-189

26. Sherief H., Abd El-Latief A.M., 2014, Application of fractional order theory of thermoelasticity to a 1D problem for a half-space, ZAMM, 94, 6, 509-515

27. Sherief H., El-Sayed A.M.A., Abd El-Latief A.M., 2010, Fractional order theory of thermoelasticity, International Journal of Solids and Structures, 47, 269-275

28. Tzou D.Y., 1995, Experimental support for the lagging behavior in heat propagation, Journal of Thermophysics and Heat Transfer, 9, 686-693

29. Youssef H.M., 2010, Theory of fractional order generalized thermoelasticity, Journal of Heat Transfer, 132, 6, 061301, 1-7

30. Wang X., Xu X., 2001, Thermoelastic waves induced by pulsed laser heating, Applied Physics A, 73, $107-114$ 